INPLASY

PROTOCOL

To cite: Almufarrij et al. Adults diagnosed with vestibular schwannomas and treated with stereotactic radiosurgery: a scoping review protocol. Inplasy protocol 2021120067. doi:

10.37766/inplasy2021.12.0067

Received: 14 December 2021

Published: 15 December 2021

Corresponding author: Ibrahim Almufarrij

ibrahim.almufarrij@manchester.ac.uk

Author Affiliation:

The University of Manchester

Support: NIHR.

Review Stage at time of this submission: Preliminary searches.

Conflicts of interest:

None declared.

\section{ADULTS DIAGNOSED WITH \\ VESTIBULAR SCHWANNOMAS AND TREATED WITH STEREOTACTIC RADIOSURGERY: A SCOPING REVIEW PROTOCOL}

\author{
Almufarrij, I1; Hannan, C22, Lloyd, S3; Munro, KJ4.
}

Review question / Objective: This review aims to catalogue and collate information on outcome measures, study designs, and dose-related changes in hearing following stereotactic radiosurgery for adults diagnosed with sporadic Vestibular Schwannoma.

Study designs to be included: Any peer-reviewed primary research publications will be eligible for inclusion.

Information sources: Electronic databases. The following databases will be systematically searched to identify relevant studies: PubMed, PsycINFO, EMBASE, EMCare, Web of Science and Cochrane Library.

INPLASY registration number: This protocol was registered with the International Platform of Registered Systematic Review and Meta-Analysis Protocols (INPLASY) on 15 December 2021 and was last updated on 15 December 2021 (registration number INPLASY2021120067). 
Schwannoma. Indeed, no scoping review has been performed to collate and report on the aforementioned areas.

Condition being studied: Vestibular Schwannomas (VS) are rare WHO Grade 1 tumours that arise from the vestibular component of the 8th cranial nerve, with a lifetime incidence of $\sim 1 / 1000$. The vast majority of these tumours are unilateral and arise sporadically, but VS may also arise as part of the tumor predisposition syndromes neurofibromatosis type 2 (NF2) and LZTR1 associated schwannomatosis. VS often present with audiological symptoms, typically hearing loss and tinnitus. Even in those patients that have functional hearing at the time of diagnosis, the great majority progress to severe hearing loss in the five years following their diagnosis. Two therapeutic strategies are currently employed in an effort to attenuate or even halt completely the progressive hearing loss experienced by the majority of patients with VS: hearing preservation surgery and stereotactic radiosurgery (SRS). SRS is potentially a very attractive treatment modality in this clinical setting as it allows for the outpatient treatment of the VS associated hearing loss in a single session, without the requirement for a general anaesthetic and with a very low risk of life-altering complications such as facial nerve palsy. Indeed, SRS practitioners in high-volume centres are increasingly making the argument that SRS should be considered as an upfront treatment at the time of diagnosis for VS patients with functional hearing, citing the excellent tumor control rates and improvement in the rates of hearing preservation when compared to the natural history. However, the data on hearing outcomes following SRS for VS are far from conclusive and are largely based on singlecentre case series or matched cohort studies, with all the attendant biases and limitations that this study design entails. Large cohort studies of patients with conservatively managed VS also report similar hearing outcomes to those reported from high-volume SRS centres. Moreover, there is a wide variation in the literature as to what is considered to be 'functional' hearing in VS, limiting the comparisons that can be made between studies in the area and making it difficult to pool the results in meta-analyses.

\section{METHODS}

Search strategy: The following databases will be systematically searched to identify relevant studies: PubMed, PsycINFO, EMBASE, EMCare, Web of Science and Cochrane Library. Backwards and forward citation tracking will be completed on eligible studies to identify additional relevant studies. No search restrictions will be imposed in terms of age, sex, publication date, status or language. However, when necessary, non-human studies will be filtered out. The review's search strategy will be developed by a medical information scientist in consultation with the review team. (("Hearing"[mesh] OR "hearing"[tw] OR "Hearing Loss"[mesh] OR "hearing loss*"[tw] OR "Hearing Disorders"[mesh] OR "deaf"[tw] OR "deafness"[tw] OR "hearing disorder*"[tw] OR "hearing problem*"[tw] OR "hearing difficult*"[tw] OR "hearing preservation*"[tw] OR "audiometry"[mesh] OR "audiometr*"[tw] OR "audiol*"[tw] OR "Hyperacusis"[tw] OR "Tinnitus"[tw]) AND ("Neuroma, Acoustic"[mesh] OR "Acoustic Neuroma*"[tw] OR "vestibular schwannoma*"[tw] OR "acoustic neurilem* "[tw] OR "vestibular neurilem*"[tw] OR "acoustic tumor*"[tw] OR "angle tumor*"[tw] OR "acoustic tumour*"[tw] OR "angle tumour*"[tw]) AND ("Radiosurgery"[Mesh] OR "Stereotaxic Techniques"[Mesh] OR "stereotactic radiosurgery"[tw] OR "radiosurgery"[tw] OR "radiosurg*"[tw] OR "cyberknife*"[tw] OR "cyberknive*"[tw] OR "gamma knife*"[tw] OR "gamma knive*"[tw] OR "cyber knife*"[tw] OR "cyber knive ${ }^{\star "}[\mathrm{tw}]$ OR "gammaknife * "[tw] OR "gammaknive*"[tw] OR "Zap-X"[tw] OR "ZapX"[tw] OR "Proton Therapy"[Mesh] OR "Proton Beam Therapy"[tw] OR "Proton Therapy"[tw])).

Participant or population: The participants of interest will be adults who were clinically 
diagnosed with VS; studies performed on children, on adults who were not diagnosed with VS or in those diagnosed with NF2 related VS will be excluded.

Intervention: The intervention of interest will be SRS; other surgical and non-surgical treatments for VS will be excluded.

Comparator: There will be no comparator of interest for this scoping review, but we will explore the correlation between radiosurgery cochlear dose and hearing.

Study designs to be included: Any peerreviewed primary research publications will be eligible for inclusion.

\section{Eligibility criteria: See above.}

Information sources: Electronic databases. The following databases will be systematically searched to identify relevant studies: PubMed, PsycINFO, EMBASE, EMCare, Web of Science and Cochrane Library.

Main outcome(s): Hearing outcomes (e.g., hearing thresholds, speech recognition thresholds and self-reported listening ability scores) will be included; other audiovestibular outcomes, including tinnitus, hyperacusis and vertigo, will be excluded, as will studies that measured only generic outcomes (e.g., quality of life).

Data management: Data management Retrieved references will be exported to a reference management tool (e.g. Mendeley) to automatically remove duplicates. Unique references will be exported to an Excel spreadsheet or an online system (e.g. Rayyan or Covidence) for eligibility screening. Selection process The title and abstract of all retrieved references will be screened for inclusion by two independent reviewers, who will then fully inspect the full text of all studies that passed the screening stage. Disagreements will be resolved by discussion or consulting another member of the review team. The selection process of this review will be presented in the PRISMA flow diagram. Data collection process and data items
The data will be extracted by one reviewer, and a proportion of the extracted data (e.g. $10-30 \%$ ) will be verified by another reviewer. Discrepancies will be resolved by discussion. All data will be extracted to a pre-designed extraction form, which includes the authors, publication year, study design, study population, hearingrelated outcomes and radiotherapy doses. When necessary, an online tool (e.g. WebPlotDigitizer) will be used to extract numerical data from graphs.

Quality assessment / Risk of bias analysis: No risk of bias assessment will be carried out as this is a scoping review.

Strategy of data synthesis: Data will be aggregated and reported narratively. The corresponding authors will be contacted to acquire missing data when needed.

Subgroup analysis: Not applicable.

Sensitivity analysis: Not applicable.

Language: No search restrictions will be imposed in terms of age, sex, publication date, status or language. However, when necessary, non-human studies will be filtered out.

Country(ies) involved: United Kingdom, Saudi Arabia.

Other relevant information: Patient and public involvement Patient and public involvement (PPI) groups were not part of the process of developing this scoping review. However, previous and related PPI work in Manchester has stressed the need to comprehensively explore and collate information on outcome measures, study designs and dose-related changes in hearing following SRS for adults diagnosed with VS in order to address the knowledge gap in this area. Furthermore, a recent consultation with the recently formed Manchester VS PPI group was characterised by a number of patients expressing their support for research into how single sided deafness associated with VS treatment could be ameliorated, as well as highlighting the impact that the hearing 
loss associated with the treatment of VS has had on their quality of life.

Keywords: Vestibular Schwannoma, stereotactic radiosurgery, Hearing, hearing preservation.

Dissemination plans: The findings of the review will be disseminated in relevant media, at conferences and in peerreviewed publications.

Contributions of each author:

Author 1 - Ibrahim Almufarrij.

Email: ibrahim.almufarrij@manchester.ac.uk

Author 2 - Cathal Hannan.

Email: cathalhannan@icloud.com

Author 3 - Simon Lloyd.

Email: sklloyd@me.com

Author 4 - Kevin J Munro.

Email: kevin.j.munro@manchester.ac.uk 